
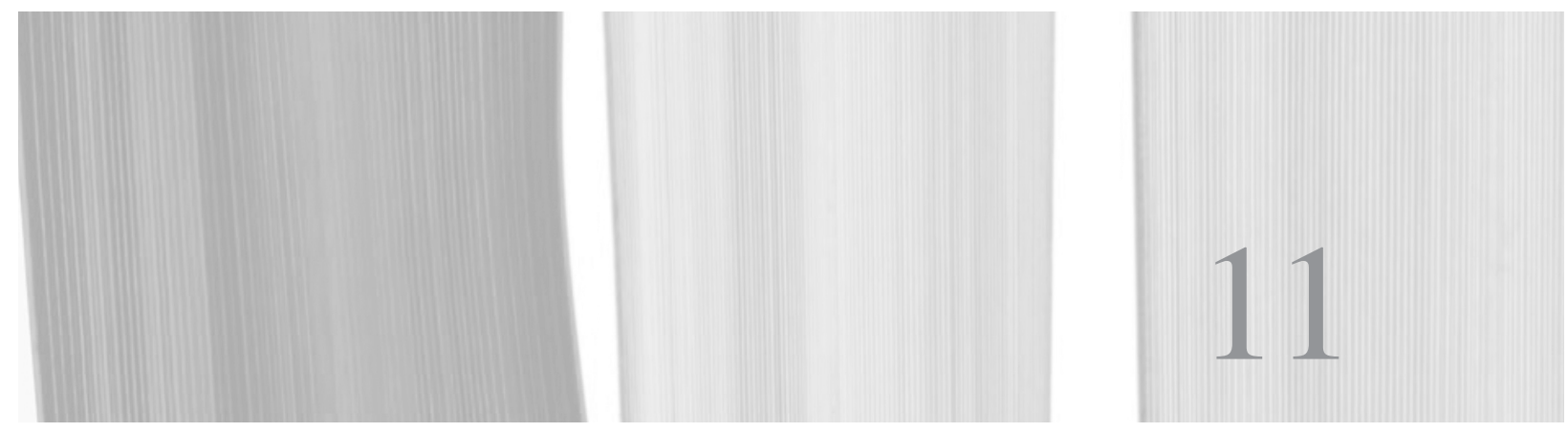

\title{
Sampling Methods for Web and E-mail Surveys
}

\author{
Ronald D. Fricker, Jr
}

\begin{abstract}
This chapter is a comprehensive overview of sampling methods for web and e-mail ('Internetbased') surveys. It reviews the various types of sampling method - both probability and nonprobability - and examines their applicability to Internet-based surveys. Issues related to Internetbased survey sampling are discussed, including difficulties assembling sampling frames for probability sampling, coverage issues, and nonresponse and selection bias. The implications of the various survey mode choices on statistical inference and analyses are summarized.
\end{abstract}

\section{INTRODUCTION}

In the context of conducting surveys or collecting data, sampling is the selection of a subset of a larger population to survey. This chapter focuses on sampling methods for web and e-mail surveys, which taken together we call 'Internet-based' surveys. In our discussion we will frequently compare sampling methods for Internet-based surveys to various types of non-Internetbased surveys, such as those conducted by postal mail and telephone, which in the aggregate we refer to as 'traditional' surveys.

The chapter begins with a general overview of sampling. Since there are many fine textbooks on the mechanics and mathematics of sampling, we restrict our discussion to the main ideas that are necessary to ground our discussion on sampling for Internet-based surveys. Readers already well versed in the fundamentals of survey sampling may wish to proceed directly to the section on Sampling Methods for Internet-based Surveys.

\section{WHY SAMPLE?}

Surveys are conducted to gather information about a population. Sometimes the survey is conducted as a census, where the goal is to survey every unit in the population. However, it is frequently impractical or impossible to survey an entire population, perhaps owing to either cost constraints or some other practical constraint, such as that it may not 
be possible to identify all the members of the population.

An alternative to conducting a census is to select a sample from the population and survey only those sampled units. As shown in Figure 11.1, the idea is to draw a sample from the population and use data collected from the sample to infer information about the entire population. To conduct statistical inference (i.e., to be able to make quantitative statements about the unobserved population statistic), the sample must be drawn in such a fashion that one can both calculate appropriate sample statistics and estimate their standard errors. To do this, as will be discussed in this chapter, one must use a probability-based sampling methodology.

A survey administered to a sample can have a number of advantages over a census, including:

- lower cost

- less effort to administer

- better response rates

- greater accuracy.
The advantages of lower cost and less effort are obvious: keeping all else constant, reducing the number of surveys should cost less and take less effort to field and analyze. However, that a survey based on a sample rather than a census can give better response rates and greater accuracy is less obvious. Yet, greater survey accuracy can result when the sampling error is more than offset by a decrease in nonresponse and other biases, perhaps due to increased response rates. That is, for a fixed level of effort (or funding), a sample allows the surveying organization to put more effort into maximizing responses from those surveyed, perhaps via more effort invested in survey design and pre-testing, or perhaps via more detailed non-response follow-up.

What does all of this have to do with Internet-based surveys? Before the Internet, large surveys were generally expensive to administer and hence survey professionals gave careful thought to how to best conduct a survey in order to maximize information accuracy while minimizing costs. However,

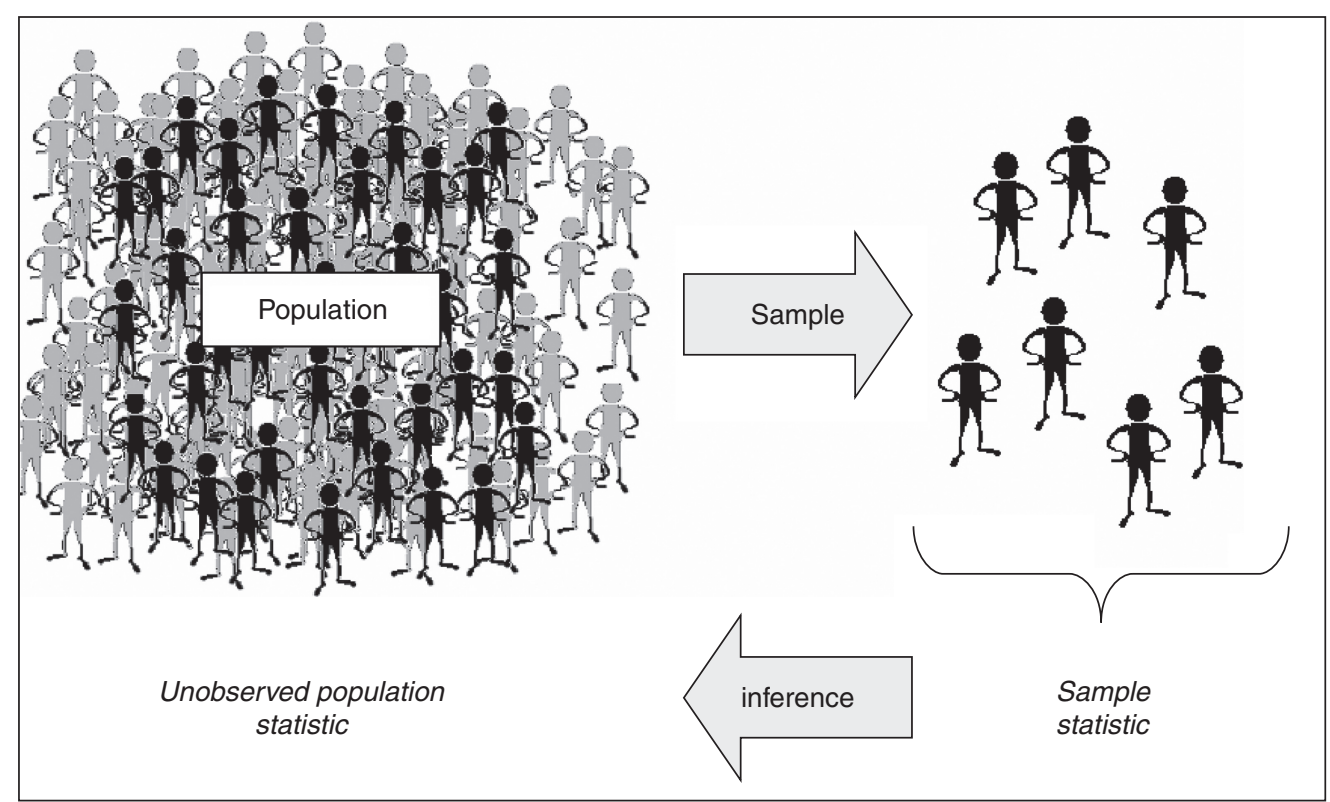

Figure 11.1 An illustration of sampling. When it is impossible or infeasible to observe a population statistic directly, data from a sample appropriately drawn from the population can be used to infer information about the population 
as illustrated in Figure 11.2, the Internet now provides easy access to a plethora of inexpensive survey software, as well as to millions of potential survey respondents, and it has lowered other costs and barriers to surveying. While this is good news for survey researchers, these same factors have also facilitated a proliferation of bad surveyresearch practice.

For example, in an Internet-based survey the marginal cost of collecting additional data can be virtually zero. At first blush, this seems to be an attractive argument in favour of attempting to conduct censuses, or for simply surveying large numbers of individuals without regard to how the individuals are recruited into the sample. And, in fact, these approaches are being used more frequently with Internet-based surveys, without much thought being given to alternative sampling strategies or to the potential impact such choices have on the accuracy of the survey results. The result is a proliferation of poorly conducted 'censuses' and surveys based on large convenience samples that are likely to yield less accurate information than a wellconducted survey of a smaller sample.
Conducting surveys, as in all forms of data collection, requires making compromises. Specifically, there are almost always tradeoffs to be made between the amount of data that can be collected and the accuracy of the data collected. Hence, it is critical for researchers to have a firm grasp of the tradeoffs they implicitly or explicitly make when choosing a sampling method for collecting their data.

\section{AN OVERVIEW OF SAMPLING}

There are many ways to draw samples from a population - and there are also many ways that sampling can go awry. We intuitively think of a good sample as one that is representative of the population from which the sample has been drawn. By 'representative' we do not necessarily mean the sample matches the population in terms of observable characteristics, but rather that the results from the data we collect from the sample are consistent with the results we would have obtained if we had collected data on the entire population.

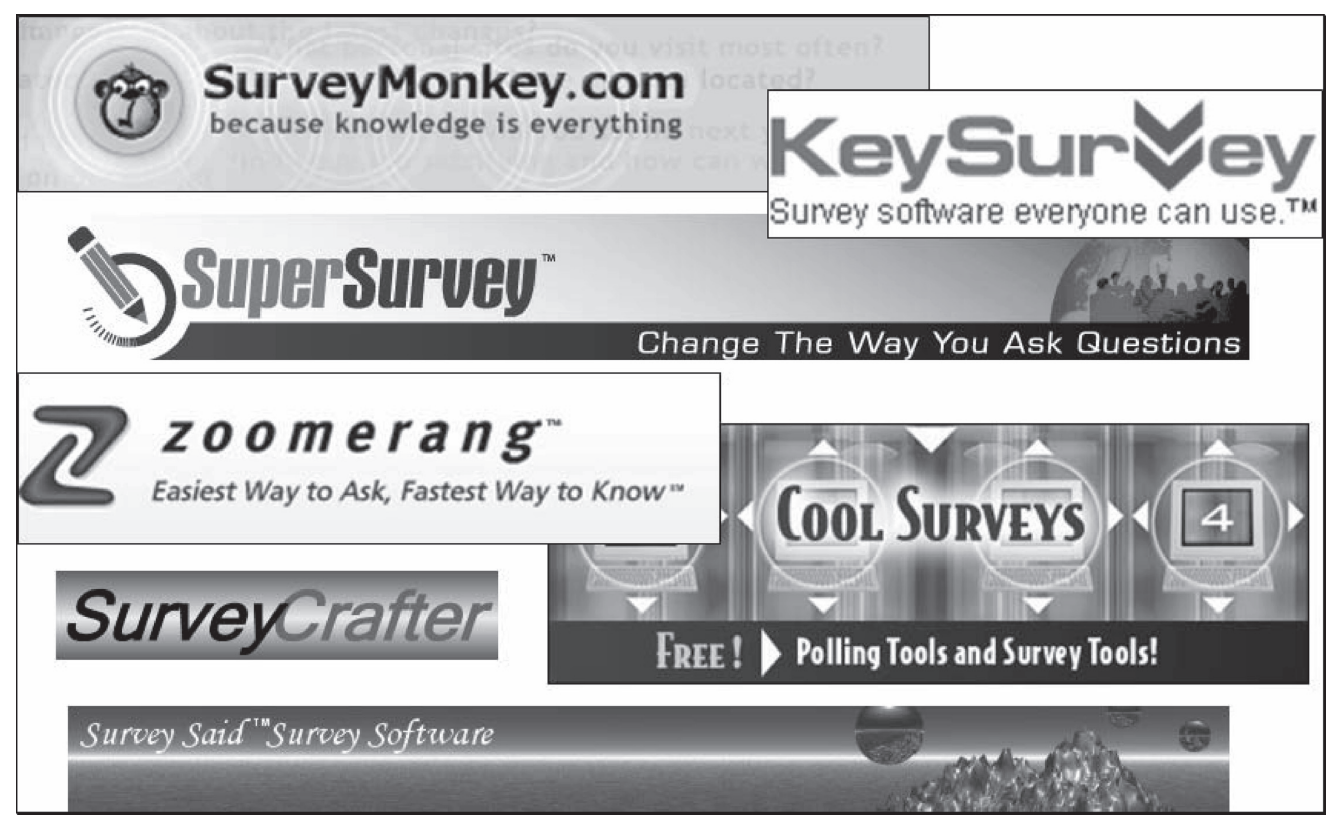

Figure 11.2 Banners for various Internet survey software (accessed January 2007) 
Of course, the phrase 'consistent with' is vague and, if this was an exposition of the mathematics of sampling, would require a precise definition. However, we will not cover the details of survey sampling here. ${ }^{1}$ Rather, in this section we will describe the various sampling methods and discuss the main issues in characterizing the accuracy of a survey, with a particular focus on terminology and definitions, in order that we can put the subsequent discussion about Internet-based surveys in an appropriate context.

\section{Sources of error in surveys}

The primary purpose of a survey is to gather information about a population. However, even when a survey is conducted as a census, the results can be affected by several sources of error. A good survey design seeks to reduce all types of error - not only the sampling error arising from surveying a sample of the population. Table 11.1 below lists the four general categories of survey error as presented and defined in Groves (1989) as part of his 'Total Survey Error' approach.

Errors of coverage occur when some part of the population cannot be included in the sample. To be precise, Groves specifies three different populations:

1 The population of inference is the population that the researcher ultimately intends to draw conclusions about.

2 The target population is the population of inference less various groups that the researcher has chosen to disregard.

3 The frame population is that portion of the target population which the survey materials or devices delimit, identify, and subsequently allow access to (Wright and Tsao, 1983).
The survey sample then consists of those members of the sampling frame that were chosen to be surveyed, and coverage error is the difference between the frame population and the population of inference.

The two most common approaches to reducing coverage error are:

- obtaining as complete a sampling frame as possible (or employing a frameless sampling strategy in which most or all of the target population has a positive chance of being sampled);

- post-stratifying to weight the survey sample to match the population of inference on some observed key characteristics.

Sampling error arises when a sample of the target population is surveyed. It results from the fact that different samples will generate different survey data. Roughly speaking, assuming a random sample, sampling error is reduced by increasing the sample size.

Nonresponse errors occur when data is not collected on either entire respondents (unit nonresponse) or individual survey questions (item nonresponse). Groves (1989) calls nonresponse 'an error of nonobservation'. The response rate, which is the ratio of the number of survey respondents to the number sampled, is often taken as a measure of how well the survey results can be generalized. Higher response rates are taken to imply a lower likelihood of nonresponse bias.

Measurement error arises when the survey response differs from the 'true' response. For example, respondents may not answer sensitive questions honestly for a variety of reasons, or respondents may misinterpret or make errors in answering questions. Measurement error is reduced in a variety of ways, including careful testing and revision of

Table 11.1 Sources of survey error according to Groves (1989)

\begin{tabular}{ll}
\hline Type of error & Definition \\
\hline Coverage & '...the failure to give any chance of sample selection to some persons in the population'. \\
Sampling & '...heterogeneity on the survey measure among persons in the population'. \\
Nonresponse & '...the failure to collect data on all persons in the sample'. \\
Measurement & '...inaccuracies in responses recorded on the survey instruments'. \\
\hline
\end{tabular}


the survey instrument and questions, choice of survey mode or modes, etc.

\section{Sampling methods}

Survey sampling can be grouped into two broad categories: probability-based sampling (also loosely called 'random sampling') and non-probability sampling. A probabilitybased sample is one in which the respondents are selected using some sort of probabilistic mechanism, and where the probability with which every member of the frame population could have been selected into the sample is known. The sampling probabilities do not necessarily have to be equal for each member of the sampling frame.

Types of probability sample include:

- Simple random sampling (SRS) is a method in which any two groups of equal size in the population are equally likely to be selected. Mathematically, simple random sampling selects $n$ units out of a population of size $N$ such that every sample of size $n$ has an equal chance of being drawn.

- Stratified random sampling is useful when the population is comprised of a number of homogeneous groups. In these cases, it can be either practically or statistically advantageous (or both) to first stratify the population into the homogeneous groups and then use SRS to draw samples from each group.

- Cluster sampling is applicable when the natural sampling unit is a group or cluster of individual units. For example, in surveys of Internet users it is sometimes useful or convenient to first sample by discussion groups or Internet domains, and then to sample individual users within the groups or domains.

- Systematic sampling is the selection of every $k$ th element from a sampling frame or from a sequential stream of potential respondents. Systematic sampling has the advantage that a sampling frame does not need to be assembled beforehand. In terms of Internet surveying, for example, systematic sampling can be used to sample sequential visitors to a website. The resulting sample is considered to be a probability sample as long as the sampling interval does not coincide with a pattern in the sequence being sampled and a random starting point is chosen.
There are important analytical and practical considerations associated with how one draws and subsequently analyzes the results from each of these types of probability-based sampling scheme, but space limitations preclude covering then here. Readers interested in such details should consult texts such as Kish (1965), Cochran (1977), Fink (2003), or Fowler (2002).

Non-probability samples, sometimes called convenience samples, occur when either the probability that every unit or respondent included in the sample cannot be determined, or it is left up to each individual to choose to participate in the survey. For probability samples, the surveyor selects the sample using some probabilistic mechanism and the individuals in the population have no control over this process. In contrast, for example, a web survey may simply be posted on a website where it is left up to those browsing through the site to decide to participate in the survey ('opt in') or not. As the name implies, such non-probability samples are often used because it is somehow convenient to do so.

While in a probability-based survey participants can choose not to participate in the survey ('opt out'), rigorous surveys seek to minimize the number who decide not to participate (i.e., nonresponse). In both cases it is possible to have bias, but in non-probability surveys the bias has the potential to be much greater, since it is likely that those who opt in are not representative of the general population. Furthermore, in non-probability surveys there is often no way to assess the potential magnitude of the bias, since there is generally no information on those who chose not to opt in.

Non-probability-based samples often require much less time and effort, and thus usually are less costly to generate, but generally they do not support statistical inference. However, non-probability-based samples can be useful for research in other ways. For example, early in the course of research, responses from a convenience sample might be useful in developing research hypotheses. Responses from convenience samples might also be useful for identifying 
issues, defining ranges of alternatives, or collecting other sorts of non-inferential data. For a detailed discussion on the application of various types of non-probability-based sampling method to qualitative research, see Patton (2002).

Specific types of non-probability samples include the following.

- Quota sampling requires the survey researcher only to specify quotas for the desired number of respondents with certain characteristics. The actual selection of respondents is then left up to the survey interviewers who must match the quotas. Because the choice of respondents is left up to the survey interviewers, subtle biases may creep into the selection of the sample (see, for example, the Historical Survey Gaffes section).

- Snowball sampling is often used when the desired sample characteristic is so rare that it is extremely difficult or prohibitively expensive to locate a sufficiently large number of respondents by other means (such as simple random sampling). Snowball sampling relies on referrals from initial respondents to generate additional respondents. While this technique can dramatically lower search costs, it comes at the expense of introducing bias because the technique itself substantially increases the likelihood that the sample will not be representative of the population.

- Judgement sampling is a type of convenience sampling in which the researcher selects the sample based on his or her judgement. For example, a researcher may decide to draw the entire random sample from one 'representative' Internet-user community, even though the population of interest includes all Internet users. Judgment sampling can also be applied in even less structured ways without the application of any random sampling.

\section{Bias versus variance}

If a sample is systematically not representative of the population of inference in some way, then the resulting analysis is biased. For example, results from a survey of Internet users about personal computer usage is unlikely to accurately quantify computer usage in the general population, simply because the sample is comprised only of those who use computers.
Taking larger samples will not correct for bias, nor is a large sample evidence of a lack of bias. For example, an estimate of average computer usage based on a sample of Internet users will likely overestimate the average usage in the general population regardless of how many Internet users are surveyed. Randomization is used to minimize the chance of bias. The idea is that by randomly choosing potential survey respondents the sample is likely to 'look like' the population, even in terms of those characteristics that cannot be observed or known.

Variance, on the other hand, is simply a measure of variation in the observed data. It is used to calculate the standard error of a statistic, which is a measure of the variability of the statistic. The precision of statistical estimates drawn via probabilistic sampling mechanisms is improved by larger sample sizes.

\section{Some important sources of bias}

Bias can creep into survey results in many different ways. In the absence of significant nonresponse, probability-based sampling is assumed to minimize the possibility of bias. Convenience sampling, on the other hand, is generally assumed to have a higher likelihood of generating a biased sample. However, even with randomization, surveys of and about people may be subject to other kinds of bias. For example, respondents may be inclined to over-or understate certain things ('sensitivity bias'), particularly with socially delicate questions (such as questions about income or sexual orientation, for example). Here we just focus on some of the more common sources of bias related to sampling.

- Frame coverage bias occurs when the sampling frame misses some important part of the population. For example, an e-mail survey using a list of e-mail addresses will miss those without an e-mail address.

- Selection bias is an error in how the individual or units are chosen to participate in the survey. It can occur, for example, if survey participation depends on the respondents having access to particular 
equipment, such as Internet-based surveys that miss those without Internet access.

- Size bias occurs when some units have a greater chance of being selected than others. For example, in a systematic sample of website visitors, frequent site visitors are more likely to get selected into the sample than those that do not. In a similar vein, when selecting from a frame consisting of e-mail addresses, individuals with multiple e-mail addresses would have a higher chance of being selected into a sample.

- Nonresponse bias occurs if those who refuse to answer the survey are somehow systematically different from those who do answer it.

\section{Historical survey gaffes}

A famous example of a survey that reached exactly the wrong inferential conclusion as a result of bias, in this case frame coverage and nonresponse bias, is the 'Literary Digest' poll in the 1936 United States presidential election. As described in Squires (1988), for their survey 'Literary Digest' assembled a sampling frame from telephone numbers and automobile registration lists. While using telephone numbers today might result in a fairly representative sample of the population, in 1936 only one in four households had a telephone and those were the more well-to-do. Compounding this, automobile registration lists only further skewed the frame towards individuals with higher incomes.

'Literary Digest' mailed 10 million strawvote ballots, of which 2.3 million were returned, an impressively large number, but it represented less than a 25 percent response rate. Based on the poll data, 'Literary Digest' predicted that Alfred Landon would beat Franklin Roosevelt 55 percent to 41 percent. In fact, Roosevelt beat Landon by 61 percent to 37 percent. This was the largest error ever made by a major poll and is considered to be one of the causes of 'Literary Digest's demise in 1938.

Gallup, however, called the 1936 presidential election correctly, even though he used significantly less data. But even Gallup, a pioneer in modern survey methods, didn't always get it right. In the 1948 United States presidential election between Harry S Truman and Thomas E. Dewey, Gallup used a quota sampling method in which each pollster was given a set of quotas of types of people to interview, based on demographics. While that seemed reasonable at the time, the survey interviewers, for whatever conscious or subconscious reason, were biased towards interviewing Republicans more often than Democrats. As a result, Gallup predicted a Dewey win of 49.5 percent to 44.5 percent: but almost the opposite occurred, with Truman beating Dewey with 49.5 percent of the popular vote to Dewey's 45.1 percent (a difference of almost 2.2 million votes). ${ }^{2}$

\section{SAMPLING METHODS FOR INTERNET-BASED SURVEYS}

This section describes specific types of Internet-based survey and the sampling methods that are applicable to each. We concentrate on differentiating whether particular sampling methods and their associated surveys allow for generalization of survey results to populations of inference or not, providing examples of some surveys that were done appropriately and well, and others that were less so. Examples that fall into the latter category should not be taken as a condemnation of a particular survey or sampling method, but rather as illustrations of inappropriate application, execution, analysis, etc. Couper (2000: 465-466) perhaps said it best,

Any critique of a particular Web survey approach must be done in the context of its intended purpose and the claims it makes. Glorifying or condemning an entire approach to survey data collection should not be done on the basis of a single implementation, nor should all Web surveys be treated as equal.

Furthermore, as we previously discussed, simply because a particular method does not allow for generalizing beyond the sample does not imply that the methods and resulting data are not useful in other research contexts.

Similarly to Couper (2000), Table 11.2 lists the most common probability and nonprobability sampling methods, and indicates which Internet-based survey mode or modes 
Table 11.2 Types of Internet-based survey and associated sampling methods

\begin{tabular}{lcc}
\hline Sampling method & Web & E-mail \\
\hline Probability-based & & $\checkmark$ \\
Surveys using a list-based sampling frame & $\checkmark$ & $\checkmark$ \\
Surveys using non-list-based random sampling & $\checkmark$ & $\checkmark$ \\
Intercept (pop-up) surveys & $\checkmark$ & $\checkmark$ \\
Mixed-mode surveys with Internet-based option & $\checkmark$ & \\
Pre-recruited panel surveys & $\checkmark$ & \\
Non-probability & & \\
Entertainment polls & $\checkmark$ & \\
Unrestricted self-selected surveys & $\checkmark$ & \\
Surveys using 'harvested' e-mail lists (and data) & $\checkmark$ & \\
Surveys using volunteer (opt-in) panels & $\checkmark$ &
\end{tabular}

may be used with each method. For example, it is possible to conduct both web and e-mail surveys using a list-based sampling frame methodology. Conversely, while it is feasible to conduct an entertainment poll by e-mail, virtually all such polls are conducted via web surveys.

\section{Surveys using a list-based sampling frame}

Sampling for Internet-based surveys using a list-based sampling frame can be conducted just as one would for a traditional survey using a sampling frame. Simple random sampling in this situation is straightforward to implement and requires nothing more than contact information (generally an e-mail address for an Internet-based survey) on each unit in the sampling frame. Of course, though only contact information is required to field the survey, having additional information about each unit in the sampling frame is desirable to assess (and perhaps adjust for) nonresponse effects.

While Internet-based surveys using listbased sampling frames can be conducted either via the web or by e-mail, if an allelectronic approach is preferred the invitation to take the survey will almost always be made via e-mail. And, because e-mail lists of general populations are generally not available, this survey approach is most applicable to large homogeneous groups for which a sampling frame with e-mail addresses can be assembled (for example, universities, government organizations, large corporations, etc). Couper (2000) calls these 'list-based samples of high-coverage populations'.

In more complicated sampling schemes, such as a stratified sampling, auxiliary information about each unit, such as membership in the relevant strata, must be available and linked to the unit's contact information. And more complicated multi-stage and cluster sampling schemes can be difficult or even impossible to implement for Internet-based surveys. First, to implement without having to directly contact respondents will likely require significant auxiliary data, which is unlikely to be available except in the case of specialized populations. Second, if (nonInternet based) contact is required, then the researchers are likely to have to resort to the telephone or mail in order to ensure that sufficient coverage and response rates are achieved.

An example of multi-stage sampling procedure, used for an Internet-based survey of realestate journalists for which no sampling frame existed, is reported by Jackob et al. (2005). For this study, the researchers first assembled a list of publications that would have journalists relevant to the study. From this list a stratified random sample of publications was drawn, separately for each of five European countries. They then contacted the managing editor at each sampled publication and obtained the necessary contact information on all of the journalists that were 'occupied with 
real-estate issues'. All of the journalists identified by the managing editors were then solicited to participate in a web survey. Jackob et al. (2005) concluded that it 'takes a lot of effort especially during the phase of preparation and planning' to assemble the necessary data and then to conduct an Internetbased survey using a multi-stage sampling methodology.

\section{Surveys using non-list-based random sampling}

Non-list-based random sampling methods allow for the selection of a probability-based sample without the need to actually enumerate a sampling frame. With traditional surveys, random digit dialing (RDD) is a non-list-based random sampling method that is used mainly for telephone surveys.

There is no equivalent of RDD for Internet-based surveys. For example, it is not possible (practically speaking) to generate random e-mail addresses (see the Issues and Challenges in Internet-based Survey Sampling section). Hence, with the exception of intercept surveys, Internet-based surveys requiring non-list-based random sampling depend on contacting potential respondents via some traditional means such as RDD, which introduces other complications and costs. For example, surveyors must either screen potential respondents to ensure they have Internet access or field a survey with multiple response modes. Surveys with multiple response modes introduce further complications, both in terms of fielding complexity and possible mode effects (again, see the Issues and Challenges in Internetbased Survey Sampling section).

\section{Intercept surveys}

Intercept surveys on the web are popup surveys that frequently use systematic sampling for every $k$ th visitor to a website or web page. These surveys seem to be most useful as customer-satisfaction surveys or marketing surveys. This type of systematic sampling can provide information that is generalizable to particular populations, such as those that visit a particular website/page. The surveys can be restricted to only those with certain IP (Internet Protocol) addresses, allowing one to target more specific subsets of visitors, and 'cookies' can be used to restrict the submission of multiple surveys from the same computer.

A potential issue with this type of survey is nonresponse. Coomly (2000) reports typical response rates in the 15 to 30 percent range, with the lowest response rates occurring for poorly targeted and/or poorly designed surveys. The highest response rates were obtained for surveys that were relevant to the individual, either in terms of the particular survey questions or, in the case of marketing surveys, the commercial brand being surveyed.

As discussed in Couper (2000), an important issue with intercept surveys is that there is no way to assess nonresponse bias, simply because no information is available on those that choose not to complete a survey. Coomly (2000) hypothesizes that responses may be biased towards those who are more satisfied with a particular product, brand, or website; towards those web browsers who are more computer and Internet savvy; and, away from heavy Internet browsers who are conditioned to ignore pop-ups. Another source of nonresponse bias for intercept surveys implemented as pop-up browser windows may be pop-up blocker software, at least to the extent that pop-up blocker software is used differentially by various portions of the web-browsing community.

\section{Pre-recruited panel surveys}

Pre-recruited panel surveys are, generally speaking, groups of individuals who have agreed in advance to participate in a series of surveys. For Internet-based surveys requiring probability samples, these individuals are generally recruited via some means other than the web or e-mail - most often by telephone or postal mail.

For a longitudinal effort consisting of a series of surveys, researchers may recruit 
panel members specifically for that effort. For smaller efforts or for single surveys, a number of companies maintain panels of individuals, pre-recruited via a probability-based sampling methodology, from which sub-samples can be drawn according to a researcher's specification. Knowledge Networks, for example, recruits all of its panel members via telephone using RDD, and it provides equipment and Internet access to those that do not have it in an attempt to maintain a panel that is a statistically valid cross section of the population (see Pineau and Dennis, 2004, for additional detail).

Pre-recruited, Internet-enabled panels can provide the speed of Internet-based surveys while simultaneously eliminating the often-lengthy recruitment process normally required. As such, they can be an attractive option to researchers who desire to field an Internet-based survey, but who require a sample that can be generalized to populations outside of the Internet-user community.

However, pre-recruited panels are not without their potential drawbacks. In particular, researchers should be aware that long-term panel participants may respond differently to surveys and survey questions than first-time participants (called 'panel conditioning' or 'time-in-sample bias'). Also, nonresponse can be an issue if the combined loss of potential respondents throughout all of the recruitment and participation stages is significant. However, as Couper (2000) concludes, '... in theory at least, this approach begins with a probability sample of the full (telephone) population, and assuming no nonresponse error permits inference to the population...'.

\section{Entertainment polls}

Internet-based entertainment polls are 'surveys' conducted purely for their entertainment value (though they are sometimes passed off to be more than what they are). On the Internet, they largely consist of websites where any visitor can respond to a posted survey. An example of an Internet-based entertainment poll is The Weekly Web Poll (www.weeklywebpoll. com). The telephone equivalent of these types of polls are call-in polls (or cell-phone textmessage polls) such as those advertised on various television shows, where viewers can vote for their favourite contestant or character. Of course, Internet-based entertainment polls are as unscientific as call-in telephone polls.

\section{Surveys using 'Harvested' e-mail lists}

Harvested e-mail lists are sets of e-mail addresses collected from postings on the web and from individuals who are (wittingly or unwittingly) solicited for their e-mail addresses. There are many commercial entities ('e-mail brokers') that sell lists of e-mail addresses or access to lists of e-mail addresses (just Google 'buy e-mail list'). Lists can also be assembled from resources on the web. For example, lists of Yahoo e-mail address holders by name or geographic area can be created by anyone via the Yahoo! People Search (http://email.people. yahoo.com/py/). Similarly, World Email.com (www.worldemail.com) has an e-mail search feature by name.

However, it is important to note that harvesting e-mail addresses and distributing unsolicited e-mail related to surveys could be a violation of professional ethical standards and/or illegal. For example, European Union Article 13(1) of the Privacy and Electronic Communications Directive prohibits the sending of unsolicited commercial e-mail. In a similar vein, the Council of American Survey Research Organizations (CASRO) Code of Standards and Ethics for Survey Research clearly states that using harvested e-mail addresses and sending unsolicited e-mail is unethical (www.casro.org/codeofstandards.cfm). For a more detailed discussion of the ethical considerations and implications, see Eynon et al., and Charlesworth (this volume), and Krishnamurthy (2002) and the references contained therein.

Samples derived from harvested e-mail lists are non-probability samples because they are based on a convenience sample of e-mail addresses. For example, Email 
Marketing Blitz (www.email-marketing-blitz. com/customized_email_list.htm) says, 'Our targeted optin [sic] email lists are updated monthly and gathered through special interest websites, entertainment websites and special alliances.' Such samples should not be confused with list-based probability samples where the e-mail addresses in the list-based sample represent a (virtually) complete list of the e-mail addresses of some target population.

The efficacy of sending unsolicited surveys to a list of purchased or otherwise procured list e-mail addresses is questionable. Not only do e-mail addresses turn over quite frequently, but many of those on the list may have been recruited either without their knowledge, or they may have inadvertently agreed by failing to uncheck a box when they signed up for something else. As a result, response rates are likely to be extremely low.

\section{Unrestricted self-selected surveys}

As with entertainment polls, unrestricted, selfselected surveys are surveys that are open to the public for anyone to participate in. They may simply be posted on a website so that anyone browsing through may choose to take the survey, or they may be promoted via website banners or other Internet-based advertisements, or they may be publicized in traditional print and broadcast media. Regardless of how they are promoted (or not), the key characteristics of these types of survey are that there are no restrictions on who can participate, and it is up to the individual to choose to participate (opt in).

For example, Berson et al. (2002) conducted a web-based survey 'to better understand the risks to adolescent girls online' by posting a link to their survey on the Seventeen Magazine Online website. Via the survey, the authors collected data on 10,800 respondents with 'identified behaviours that put them at risk'. The researchers were careful to appropriately qualify their results:

The results highlighted in this paper are intended to explore the relevant issues and lay the groundwork for future research on youth in cyberspace. This is considered an exploratory study which introduces the issues and will need to be supplemented with ongoing research on specific characteristics of risk and prevention intervention. Furthermore, the generalizability of the study results to the larger population of adolescent girls needs to be considered. Due to anonymity of the respondents, one of the limitations of the research design is the possibility that the survey respondents did not represent the experience of all adolescent girls or that the responses were exaggerated or misrepresented.

Unrestricted, self-selected surveys are a form of convenience sampling and, as such, the results cannot be generalized to a larger population. But as Berson et al. illustrate, that does not necessarily negate their usefulness for research.

The web can also facilitate access to individuals who are difficult to reach either because they are hard to identify, locate, or perhaps exist in such small numbers that probability-based sampling would be unlikely to reach them in sufficient numbers. Coomber (1997) describes such a use of the web for fielding a survey to collect information from drug dealers about drug adulteration/dilution. By posting invitations to participate in a survey on various drugrelated discussion groups, Coomber collected data from 80 survey respondents (that he deemed reliable) located in 14 countries on four different continents. The sample was certainly not generalizable, but it also provided data that was unlikely to be collected in any other way, and which Coomber found consistent with other research.

In addition, Alvarez et al. (2002) proposed that these types of non-probability sample can be useful and appropriate for conducting experiments (say, in the design of web pages or web surveys) by randomly assigning members of the sample to control and experimental groups. In terms of psychology experiments, Siah (2005) states, 'For experimental research on the Internet, the advantage of yielding a heterogeneous sample seems persuasive considering that the most common criticism of psychological research is its over-reliance on college student samples.' 


\section{Volunteer (opt-in) panels}

Volunteer (opt-in) panels are similar in concept to the pre-recruited panels, except the volunteers are not recruited using a probability-based method. Rather, participants choose to participate, perhaps after coming across a solicitation on a website. In this regard, volunteer panels are similar to unrestricted, self-selected surveys except that those who opt in do so to take a continuing series of surveys. Harris Interactive manages such a volunteer panel. Its website (www.harrispollonline.com/became.asp) states, 'You may have become a member of the Harris Poll Online in one of several ways:

- By registering directly with us through our website (http://www.harrispollonline.com); or

- By opting in to participate in the Harris Poll Online as a result of an offering made in conjunction with one of our many online partners.'

Often these panels are focused on market research, soliciting consumer opinions about commercial products, and participants sometimes do it for monetary incentives. For example, the Harris website states,

We offer the opportunity to earn HIPoints for the majority of our studies. On occasion a study will be conducted that will not have HIPoints associated with it, but this only occurs in exceptions. Once you've accumulated enough points you may redeem them for your choice of a variety of great rewards (www.harrispollonline.com/benefit.asp).

\section{ISSUES AND CHALLENGES IN INTERNET-BASED SURVEY SAMPLING}

All survey modes have their strengths and weaknesses; Internet-based surveys are no different in this regard. The various strengths and weaknesses are more or less important, depending on the survey's purpose. Drawing an appropriate sample that will provide the data necessary to appropriately address the research objective is critical. Hence, in this section we focus on the issues and challenges related to sampling for Internet-based surveys.

\section{Sampling Frame and Coverage Challenges}

A frequent impediment for conducting largescale, Internet-based surveys is the lack of a sampling frame. Simply put, no single registry or list of e-mail addresses exists and thus listbased sampling frames are generally available only for specific populations (government organizations, corporations, etc).

Compounding this difficulty, and leaving aside the issue of population coverage to be discussed shortly, it is impossible to employ a frameless sampling strategy, since for all practical purposes one cannot assemble random e-mail addresses. Of course, it is theoretically possible to 'construct' e-mail addresses by repeatedly randomly concatenating letters, numbers, and symbols, but the sheer variety of e-mail addresses means most of the constructed addresses will not work. More importantly, the unstructured nature of the Internet means that even if one could tolerate the multitude of undeliverable e-mail messages that would result, they would not be useful as the basis for a probability sample.

In terms of coverage, it is widely recognized that Internet-based surveys using only samples of Internet users do not generalize to the general public. While Internet penetration into households continues at a rapid pace, the penetration is far from complete (compared to, say, the telephone) and varies widely by country and region of the world. ${ }^{3}$ The point is, if the target of inference is the general public, considerable coverage error remains for any sample drawn strictly from Internet users.

Now, even if there is minimal coverage error for a particular Internet-based survey effort, when using only an Internet-based survey mode the target population must also be sufficiently computer-literate and have both regular and easy access to the Internet to facilitate responding to the survey. Simply put, just because an organization maintains a 
list of e-mail addresses for everyone in the organization it does not necessarily follow that every individual on the list has equal access. Lack of equal access could result in significant survey selection and nonresponse biases.

\section{Mixed-mode surveys using internet-based and traditional media}

For some surveys it may be fiscally and operationally possible to contact respondents by some mode other than e-mail, such as mail or telephone. In these cases the survey target population can be broader than that for which an e-mail sampling frame is available, up to and including the general population. But at present such a survey must also use multiple survey modes to allow respondents without Internet access the ability to participate. Mixed-mode surveys may also be useful for alleviating selection bias for populations with uneven or unequal Internet access, and the sequential use of survey modes can increase response rates.

For example, Dillman (2007: 456) describes a study in which surveys that were fielded using one mode were then followed up with an alternate mode three weeks later. As shown in Table 11.3, in all cases the response rate increased after the follow-up. Now, of course, some of this increase can be attributed simply to the fact that a follow-up effort was conducted. However, the magnitude of the increases also suggests that offering a different response mode in the follow-up could be beneficial.

However, mixed-mode surveys are subject to other issues. Two of the most important are mode effects and respondent mode preferences. Mode effects arise when the type of survey affects how respondents answer questions. Comparisons between Internet-based surveys and traditional surveys have found conflicting results, with some researchers reporting mode effects and others not. See, for example, the discussion and results in Schonlau et al. (2004: 130). Though not strictly a sampling issue, the point is that researchers should be prepared for the existence of mode effects in a mixed-mode survey. Vehovar and Manfreda's overview chapter (this volume) explores in greater detail the issues of combining data from Internetbased and traditional surveys.

In addition, when Internet-based surveys are part of a mixed-mode approach, it is important to be aware that the literature currently seems to show that respondents will tend to favour the traditional survey mode over an Internet-based mode. See, for example, the discussions in Schonlau et al. (2002) and Couper (2000: 486-487). Fricker and Schonlau (2002), in a study of the literature on web-based surveys, found 'that for most of the studies respondents currently tend to choose mail when given a choice between web and mail. In fact, even when respondents are contacted electronically it is not axiomatic that they will prefer to respond electronically'.

The tendency to favour non-Internet-based survey modes lead Schonlau et al. (2002: 75) to recommend for mixed-mode mail and web surveys that:

... the most effective use of the Web at the
moment seems to involve a sequential fielding
scheme in which respondents are first encouraged
to complete the survey via the Web and then
nonrespondents are subsequently sent a paper
survey in the mail. This approach has the advantage
of maximizing the potential for cost savings from

Table 11.3 As reported in Dillman (2007), using an alternate survey mode as a follow-up to an initial survey mode can result in higher overall response rates

\begin{tabular}{lll}
\hline $\begin{array}{l}\text { Initial survey mode and } \\
\text { response rate }\end{array}$ & $\begin{array}{l}\text { Follow-up survey mode and combined } \\
\text { response rate }\end{array}$ & Response rate increase \\
\hline Mail $(75 \%)$ & Telephone $(83 \%)$ & $8 \%$ \\
Telephone $(43 \%)$ & Mail $(80 \%)$ & $37 \%$ \\
IVR $^{5}(28 \%)$ & Telephone $(50 \%)$ & $22 \%$ \\
Web (13\%) & Telephone (48\%) & $35 \%$ \\
\hline
\end{tabular}


using Internet while maintaining the population coverage and response rates of a mail survey.

\section{Web-based recruitment issues and effects}

Whether e-mail addresses are constructed, assembled from third-party sources, or harvested directly from the web, there is the issue of unsolicited survey e-mail as spam. For example, Sheehan (1999) conducted a survey with e-mail addresses harvested from www.Four11.com and stated, 'Several individuals receiving the solicitation e-mail censured the researchers for sending out unsolicited e-mails, and accused the researchers of "spamming".' They further recounted that 'One [ISP] system operator [who observed a large number of e-mail messages originating from a single address] then contacted his counterpart at our university.'

In addition, distributing an unsolicited Internet-based survey is also not without its perils. For example, Andrews et al. (2002) report on a study of 'hard-to-involve Internet users' - those who lurk in, but do not participate publicly in, online discussion forums. In their study, an invitation to participate in a web survey was posted as a message to 375 online community discussion boards. While they collected 1,188 valid responses (out of 77,582 discussion board members), they also 'received unsolicited email offers, some of which were pornographic in content or aggressive in tone' and they had their web server hacked twice, once with the infection of a virus.

In spite of the challenges and possible perils, it is possible to recruit survey participants from the web. For example, Alvarez et al. (2002) conducted two Internet-based recruitment efforts - one using banner advertisements on web pages and another using a subscription check box. In brief, their results were as follows.

- In the first recruitment effort, Alvarez et al. ran four 'banner' campaigns in 2000 with the intention of recruiting survey participants using web-page banner advertisements. In the first campaign, which is representative of the other three, an animated banner advertisement resulted in more than 3.5 million 'impressions' (the number of times the banner was displayed), which resulted in the banner being clicked 10,652 times, or a rate of 3 clicks per 1,000 displays. From these 10,652 clicks, 599 survey participants were recruited.

- In the second recruitment effort, the authors ran a 'subscription' campaign in 2001 in which they arranged with a commercial organization to have a check box added to subscription forms on various websites. Essentially, Internet users who were registering for some service were given an opportunity to check a box on the service's subscription form indicating their willingness to participate in a survey. As part of this effort, the authors conducted two recruitment drives, each of which was intended to net 10,000 subscriptions. Across the two campaigns, 6,789 new survey participants were obtained from 21,378 subscribers.

The good news from the Alvarez et al. (2002) study is that, even though the banner approach yielded fewer new survey participants, both methods resulted in a significant number of potential survey respondents over a relatively short period of time 3,431 new subjects over the course of six or seven weeks from the banner campaigns, and 6,789 new subjects over the course of three weeks from the subscription campaigns. Each banner subject cost about \$7.29 to recruit, while the subscription subjects cost only $\$ 1.27$ per subject. (Unfortunately, the authors did not present any data on survey completion rates, so we do not know whether there were differences between the two samples that might have favored one over the other).

The bad news is that the two groups differed significantly in all of the demographic categories collected (gender, age, race, and education) and they differed in how they answered questions on exactly the same survey. In addition, both groups differed significantly from the demographics of the Internet population as measured by the August 2000 Current Population Survey. The problem, of course, is that there are clear effects associated with how subjects 
are recruited, such that the resulting samples are different even from the general Internet population. Shillewaert et al. (1998) found similar recruitment method biases. Hence, while it is possible to ethically recruit survey participants from the web, it seems that the recruitment methodology affects the types of individual that self-select into the sample.

\section{Improving response rates for Internet-based surveys}

Response rates have a direct effect on sampling: the higher the response rate, the fewer people need to be sampled to achieve a desired number of survey completions. In addition, higher response rates are associated with lower nonresponse bias.

Unfortunately, in a summary of the academic survey-related literature up through 2001, Fricker and Schonlau (2002) concluded that 'Web-only research surveys have currently only achieved fairly modest response rates, at least as documented in the literature.' S. Fricker et al. (2005) similarly summarized the state of affairs as 'Web surveys generally report fairly low response rates.'

A good illustration of this is the Couper et al. (1999) study in which employees of five US federal government statistical agencies were randomly given a mail or e-mail survey. Comparable procedures were used for both modes, yet higher response rates were obtained for mail (68-76 percent) than for e-mail (37-63 percent) across all of the agencies.

Incentives are a common and effective means for increasing response rates in traditional surveys. Goritz (2006) is an excellent review of the use of incentives in survey research in which he distinguishes their use in traditional surveys from Internet-based surveys and provides a nice discussion of the issues associated with using incentives in Internet-based surveys. Open issues include:

- how best to deliver an incentive electronically;

- whether it is better to provide the incentive prior to a respondent taking the survey or after;
- whether incentives have different effects for individuals taking a survey one time versus prerecruited panel members who take a series of surveys.

Individual studies of Internet-based surveys have generally found incentives to have little or no effect. For example, Coomly (2000) found that incentives had little effect on response rates for pop-up surveys, and Kypri and Gallagher (2003) found no effect in a web-based survey. However, Göritz (2006) conducted a meta-analysis of 32 experiments evaluating the impact of incentives on survey 'response' (the fraction of those solicited to take the survey that actually called up the first page of the survey), and 26 experiments evaluating the effect of incentives on survey 'retention' (the fraction of those who viewed the first page that actually completed the survey). From the meta-analysis, Görtiz concluded that 'material incentives promote response and retention in Web surveys' where 'material incentives increase the odds of a person responding by $19 \%$ over the odds without incentives' and 'an incentive increased retention by $4.2 \%$ on average'.

In addition to incentives, Dillman (2007) and Dillman et al. (1999) have put forward a number of survey procedural recommendations to increase survey response rates, based on equivalent methods for traditional surveys, which we will not re-cover here since they are mainly related to survey design and fielding procedures. While we do note that the recommendations seem sensible, Couper (2000) cautions that 'there is at present little experimental literature on what works and what does not'.

\section{Bigger samples are not always better}

With Internet-based surveys using a listbased sampling frame, rather than sending the survey out to a sample, researchers often simply send the survey out to the entire sampling frame. That is, researchers naively conducting (all electronic) Internet-based surveys - where the marginal costs for additional surveys can 
be virtually nil - often fail to recognize the 'trade-off between easy, low cost access to large numbers of patients [participants] and the representativeness in the population being studied' (Soetikno et al., 1997). As we previously discussed, for both probability and non-probability-based samples, larger sample sizes do not necessarily mean the sample is more representative of any greater population: a sample can be biased whether it is large or small.

One might argue that in these situations the researchers are attempting to conduct a census, but in practice they are forgoing a probability sample in favour of a convenience sample by allowing members of the sampling frame to opt into the survey. Dillman et al. (1999) summarized this practice as follows: '...the ease of collecting hundreds, thousands, or even tens of thousands of responses to web questionnaires at virtually no cost, except for constructing and posting, appears to be encouraging a singular emphasis on the reduction of sampling error'. By this Dillman et al. mean that researchers who focus only on reducing sampling error by trying to collect as large a sample as possible miss the point that it is equally important to reduce coverage, measurement, and nonresponse error in order to be able to accurately generalize from the sample data.

A myopic focus on large sample sizes and the idea that large samples equate to sample representativeness which equates to generalizability - occurs with convenience sample-based web and e-mail surveys as well. 'Survey2000' is an excellent example of this type of focus. A large-scale, unrestricted, self-selected survey, conducted as a collaborative effort between the National Geographic Society (NGS) and some academic researchers, Survey2000 was fielded in 1998. The survey was posted on the National Geographic Society's website and participants were solicited both with a link on the NGS homepage and via advertisements in NGS periodicals, other magazines, and newspapers.

Upon completion of the effort, Witte et al. (2000) report that more than 80,000 surveys were initiated and slightly more than 50,000 were completed. While this is an impressively large number of survey completions, the unrestricted, self-selected sampling strategy clearly results in a convenience sample that is not generalizable to any larger population. Yet, Witte et al. (2000) go to extraordinary lengths to rationalize that their results are somehow generalizable, while simultaneously demonstrating that the results of the survey generally do not correspond to known population quantities.

\section{Misrepresenting convenience samples}

A related and significant concern with nonprobability-based sampling methods, both for Internet-based and traditional surveys, is that survey accuracy is characterized only in terms of sampling error and without regard to the potential biases that may be present in the results. While this has always been a concern with all types of survey, the ease and spread of Internet-based surveys seems to have exacerbated the practice. For example, the results of an 'E-Poll' were explained as follows:

THE OTHER HALF / E-Poll $®$ Survey of 1,007 respondents was conducted January 16-20, 2003. A representative group of adults $18+$ were randomly selected from the E-Poll online panel. At a $95 \%$ confidence level, a sample error of $+/-3 \%$ is assumed for statistics based on the total sample of 1,007 respondents. Statistics based on sub-samples of the respondents are more sensitive to sampling error. (From a press release posted on the E-Poll website.)

No mention was made in the press release that the 'E-Poll online panel' consists of individuals who had chosen to participate in online polls, nor that they were unlikely to be representative of the general population. Rather, it leaves readers with an incorrect impression that the results apply to the general population when, in fact, the margin of error for this particular survey is valid only for adult members of that particular E-Poll online panel. 
In response to the proliferation of these types of misleading statement, the American Association for Public Opinion Research (AAPOR) has publicly stated that 'The reporting of a margin of sampling error associated with an opt-in or self-identified sample (that is, in a survey or poll where respondents are self-selecting) is misleading.' They go on to say, 'AAPOR considers it harmful to include statements about the theoretical calculation of sampling error in descriptions of such studies, especially when those statements mislead the reader into thinking that the survey is based on a probability sample of the full target population' (AAPOR, 2007).

\section{IN SUMMARY}

A useful way to summarize which sampling methods apply to which types of Internetbased survey is to group them by respondent 'contact mode'. That is, every survey effort can be classified according to how the respondents are contacted (the contact mode), how they are asked to complete the survey (the response mode), and then how subsequent communication is conducted (the followup mode). Each of these can be executed in different media, where the media are telephone, mail, web, e-mail, and so forth. For example, respondents may be contacted by telephone to participate in a web survey with follow-up done by mail.

Explicitly specifying contact, response, and follow-up modes is often irrelevant for traditional surveys, since respondents that have been asked to take, say, a telephone survey have generally been contacted via the same mode. While not a strict rule - for example, a telephone survey may be preceded by mailed invitation to each survey respondent - it is often the case. In comparison, given the challenges that we have discussed in this chapter, the contact, response, and follow-up modes are much more likely to differ with Internet-based surveys.

In terms of sampling for Internet-based and e-mail surveys, what is relevant is that the sampling methodology is generally driven by the contact mode, not the response mode. Hence, as shown in Table 11.4, we can organize sampling strategies by contact mode, where the check marks indicate which sampling strategies are mainly associated with the various contact methods.

Note that we are focusing explicitly on an Internet-based survey response mode in Table 11.4. So, for example, while systematic sampling can be applied to phone or mail surveys, the telephone is not likely to be used as a contact medium for an Internetbased survey using systematic sampling, and hence those cells in the table are not checked. Similarly, while there is a plethora of phonein entertainment polls, neither the telephone nor postal mail is used to contact respondents to take Internet-based entertainment polls.

From Table 11.4 we can broadly summarize the current state of the art for the various Internet-based survey methods and their limitations as follows.

- Entirely web-based surveys, meaning surveys in which the potential respondents are contacted on the web and take a web survey, are chiefly limited to collecting data from non-probabilitybased samples.

- The exception is systematic sampling for popup/intercept surveys that are predominantly used for customer-satisfaction types of survey associated with specific websites or web pages.

- Respondent contact for Internet-based surveys using non-probability samples can also be conducted via traditional (non-Internet-based) media and advertising

- Research surveys that require probability sampling are very limited when using an Internet-based contact mode (web and e-mail).

- E-mail is useful as a contact mode only if a list of e-mail addresses is available. Such a list is an actual or de facto sampling frame, from which a sample may be drawn or a census attempted.

- The population of inference is usually quite limited when using an e-mail address sampling frame. It is generally the sampling frame itself.

- A poorly conducted census of an entire e-mail list may limit the survey results even further, since nonresponse and other biases may 
Table 11.4 Sampling strategies for Internet-based surveys by contact mode

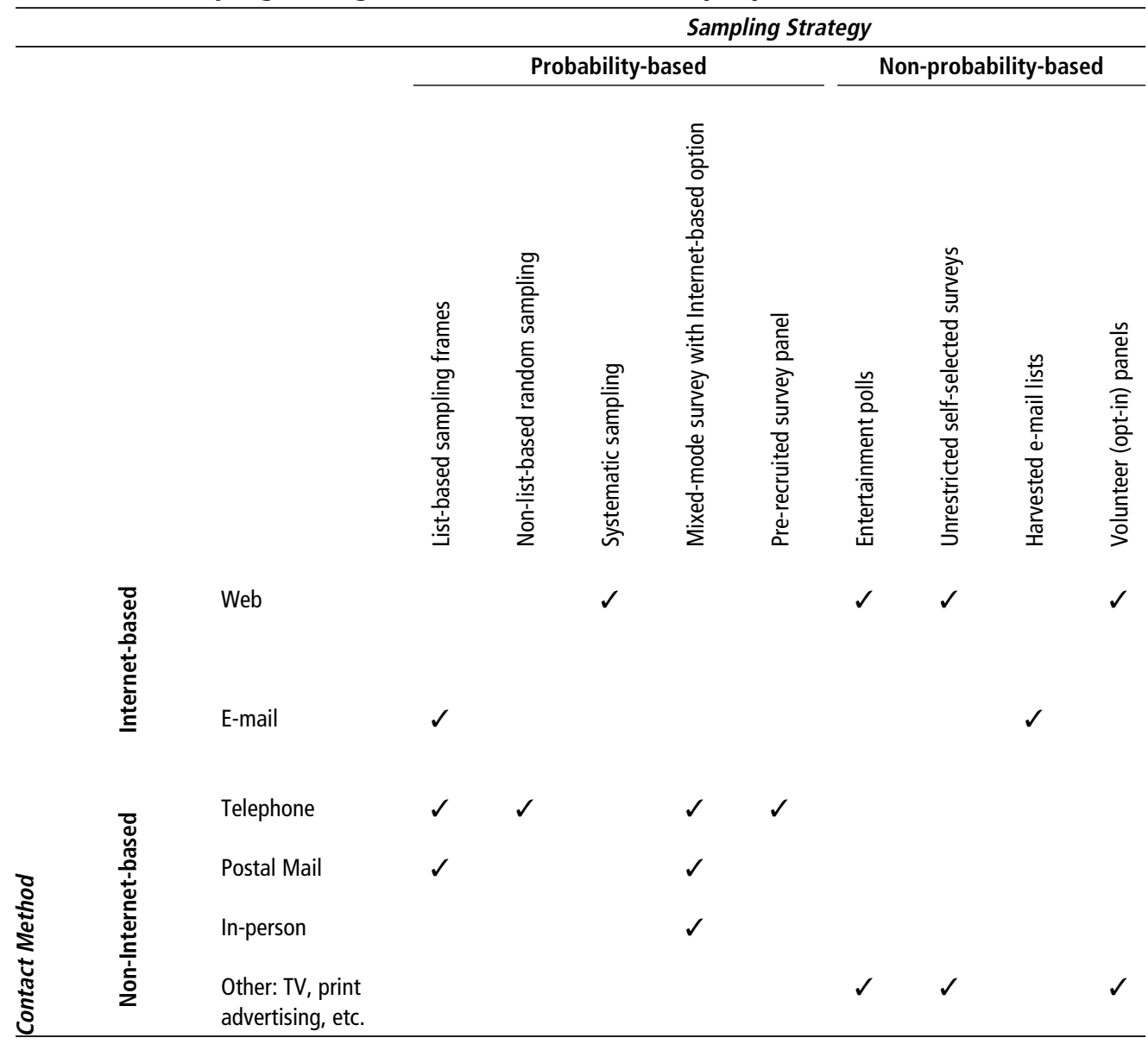

preclude generalizing even to the sample frame.

- If the research objectives require inferring from the survey results to some general population, then respondents will most likely have to be contacted by a non-Internet-based medium.

- If the population of inference is a population in which some of the members do not have e-mail/web access, then the contact mode will have to be a non-Internet-based medium.

- Under such conditions, the survey will have to be conducted using a mixed mode, so that those without Internet access can participate. Conversely, lack of a non-Internet-based survey mode will result in coverage error with the likely consequence of systematic bias.

- Pre-recruited panels can provide ready access to pools of Internet-based survey respondents, but to allow generalization to some larger, general population such panels need to be recruited using probability sampling methods from the general population (usually via RDD). And, even under such conditions, researchers need to carefully consider whether the panel is likely to be subject to other types of bias.

\section{Looking to the future}

So, what does the future hold for Internetbased survey sampling? At this point in the Internet's development, with its rapid expansion and continued evolution, it's truly impossible to say. Yet we can hazard a few guesses.

First, if the Internet continues to expand but largely maintains its current structure, then advances in sampling methods that will 
allow random sampling of, and inference to, general populations will be at best slow and difficult to develop. This follows from the fact that Internet-wide sampling frames are simply unavailable under the current Internet structure/organization, and no general frameless sampling strategies yet exist. Unless the way the Internet is organized and operated changes, it seems this will continue to be the case into the foreseeable future.

That said, survey sampling methodologists should endeavour to develop new sampling paradigms for Internet-based surveys. The fundamental requirement for a probabilitybased sampling scheme is that every member of the target population has a known, non-zero probability of being sampled. While in traditional surveys this can be achieved via various frame and frameless sampling strategies, it does not necessarily follow that Internet surveys must use those same sampling strategies. Rather, new sampling methods that take advantage of the unique characteristics of the Internet, such as the near-zero marginal cost for contacting potential respondents, should be explored and developed.

In addition, researchers considering conducting an Internet-based survey should consider whether the capabilities of the web can be leveraged to collect the desired data in some other innovative fashion. For example, Lockett and Blackman (2004) present a case study of Xenon Laboratories, an Internet-based financial services firm that employed a novel approach to market research. Xenon Laboratories wanted to collect data on foreignexchange charges by credit-card companies on business travellers. They recognized that neither the travellers nor the creditcard companies were likely to respond to a survey on this topic, whether fielded over the web or otherwise. Instead Xenon Laboratories developed the Travel Expenses Calculator (www.xe.com/tec) and the Credit Card Charges Calculator (www.xe.com/ccc) and posted them on the web for anyone to use for free. These tools help foreign business travellers to accurately calculate the cost of a business expense receipt in terms of their own currency.
Lockett and Blackman (2004) say, 'On the basis of this information [input by those using the calculators] it is possible to conduct basic market research by aggregating the inputted calculations. Xenon is now in the unique position to analyse whether or not the different card providers employ the same charging levels and whether or not these companies' charge structures vary according to geographical region.' They go on to conclude, 'This value-added approach, which is mutually beneficial to both parties, is an important and novel approach to market research.'

Second, it is also possible that technological innovation will facilitate other means of sampling for Internet-based surveys (and for conducting the surveys themselves, for that matter). For example, current trends seem to point towards a merging of the Internet with traditional technologies such as television and telephone. It is quite possible that sometime in the future all of these services will merge into one common household device through which a consumer could simultaneously watch television, surf the web, send e-mail, and place telephone calls. Depending on how this evolves, various types of random sampling methodologies, as well as new survey modes, may become possible. For example, it may become feasible, and perhaps even desirable, to sample respondents via RDD (or something very similar), have an interviewer call the respondent, and then in real time present the respondent with an interviewer-assisted, web-based survey.

It is also possible that, as the technology matures, these combined television-Internettelephone appliances will become as ubiquitous as televisions are today, with service offered only by a few large companies. If so, then it may ultimately be possible to use the companies' subscriber listings as sampling frames (much as telephone directories were used pre-RDD in the mid-1900s). Or it may be that some other state emerges that lends itself to some form of sampling that is not possible today. The point is that the Internet is still very much in its infancy, and the current difficulties surrounding sampling 
for Internet-based survey described in this chapter may or may not continue into the future.

To put this in a historical context, note that while the telephone was invented in the late 1800s, and telephone systems developed and expanded rapidly through the early 1900 s, it was not until the mid-1900s that telephone coverage was sufficiently large and standards for telephone numbers adopted that made RDD possible. In fact, the foundational ideas for an efficient RDD sampling methodology were not proposed until the early 1960s (Cooper, 1964), after which it took roughly another decade of discussion and development before RDD as we know it today became commonplace. ${ }^{4}$ Thus, in total, it was roughly a century after the invention of the telephone before RDD became an accepted sampling methodology.

In comparison, the web has been in existence, in a commercial sense, only for little more than a decade or two. As with the telephone in the late 1800s and early 1900s, we are in a period of technological innovation and expansion with the Internet. However, unlike the telephone, given today's pace of innovation, the Internet and how we use it is likely to be quite different even just a few years from now. How this affects sampling for Internet-based surveys remains to be seen.

\section{NOTES}

1 Readers interested in the mathematics should consult one of the classic texts such as Kish (1965) or Cochran (1977); readers interested in a summary treatment of the mathematics and/or a more detailed discussion of the sampling process may consult a number of other texts, such as Fink (2003) or Fowler (2002). For those specifically interested in sampling methods for qualitative research, see Patton (2002).

2 These were not the only errors made in the 1936 and 1948 US presidential election polls (for more detail, see Zetterberg (2004) or Ross (1977)), but they were significant errors, and are highlighted here to illustrate that the various biases that can creep into survey and data collection can be both subtle and non-trivial.

3 For example, as of January 2007 Internet World Stats (2007) reported that 35 countries had greater than 50 percent Internet penetration, ranging from a high of 86.3 percent for Iceland to 50 percent for the Czech Republic. In comparison, Internet penetration for the rest of the world was estimated to be 8.7 percent.

4 See, for example, 'Random Digit Dialing as a Method of Telephone Sampling (Glasser and Metzger, 1972), 'An Empirical Assessment of Two Telephone Sampling Designs (Groves, 1978), and 'Random Digit Dialing: A Sampling Technique for Telephone Surveys' (Cummings, 1979).

5 IVR stands for Interactive Voice Response. These are automated telephone surveys in which prerecorded questions are used and respondents' answers are collected using voice-recognition technology.

\section{REFERENCES}

Alvarez, R.M., Sherman, R.P. and VanBeselaere, C. (2002) 'Subject acquisition for web-based surveys', dated September 12, 2002, accessed on-line at survey.caltech.edu/alvarez.pdf on September 29, 2006.

American Association for Public Opinion Research (AAPOR) (2007) 'Reporting of margin of error or sampling error in online and other surveys of self-selected individuals'. Accessed online at www. aapor.org/pdfs/2006/samp_err_stmt.pdf on April 23, 2007.

Andrews, D., Nonnecke, B. and Preece, J. (2002) 'Electronic survey methodology: a case study in reaching hard-to-involve Internet users', International Journal of Human-Computer Interaction, 12 (2): 185-210.

Berson, I.R., Berson, M.J. and Ferron, J.M. (2002) 'Emerging risks of violence in the digital age: lessons for educators from an online study of adolescent girls in the United States', Journal of School Violence, 1(2), 51-72. Published simultaneously online in Meridian, A Middle School Computer Technologies Journal, accessed at www2.ncsu.edu/unity/lockers/project/ meridian/sum2002/cyberviolence/cyberviolence.pdf on September 29, 2006.

Cochran, William G. (1977) Sampling Techniques. New York: John Wiley.

Comley, P. (2000) 'Pop-up surveys: what works, what doesn't work and what will work in the future.' Proceedings of the ESOMAR worldwide Internet conference Net Effects 3, volume 237. Amsterdam, $\mathrm{NL}$ : ESOMAR. Accessed at www.virtualsurveys.com/ news/papers/paper_4.asp on September 21, 2006.

Coomber, R. (1997) Using the Internet for Survey Research, Sociological Research Online, 2, 14-23. 
Cooper, S.L. (1964) 'Random sampling by telephone: an improved method', Journal of Marketing Research, 1 (4): 45-8.

Couper, Mick P. (2000) 'Review: web surveys: a review of issues and approaches', The Public Opinion Quarterly, 64 (4): 464-94.

Couper, Mick P., Blair, J. and Triplett, T. (1999) 'A comparison of mail and e-mail for a survey of employees in federal statistical agencies', Journal of Official Statistics, 15 (1): 39-56.

Cummings, K.M. (1979) 'Random Digit Dialing: a sampling technique for telephone surveys', Public Opinion Quarterly, 43, 233-44.

Dillman, D.A. (2007) Mail and Internet Surveys: The Tailored Design Method (2007 update with new Internet, visual, and mixed-mode guide), 2nd edn. New York: John Wiley.

Dillman, D.A., Tortora, R.D. and Bowker, D. (1999) 'Principles for constructing web surveys', accessed online at www.sesrc.wsu.edu/dillman/ papers/websurveyppr.pdf on January 27, 2005.

Fink, Arlene (2003) How to Sample in Surveys, 2nd edn: The Survey Kit, volume 7. Thousand Oaks: Sage.

Fowler, Jr. and Floyd J. (2002) Survey Research Methods, 3rd edn: Applied Social Research Methods Series, volume 1. Thousand Oaks: Sage.

Fricker, Jr., R.D. and Schonlau, M. (2002) 'Advantages and disadvantages of Internet research surveys: evidence from the literature', Field Methods, 14: 347-67.

Fricker, S., Galesic, M., Tourangeau, R. and Yan, T. (2005) 'An experimental comparison of web and telephone surveys', Public Opinion Quarterly, 69 (3): 370-92.

Glasser, G.J. and Metzger, G.D. (1972) 'Random-Digit Dialing as a method of telephone sampling', Journal of Marketing Research, 9 (1), 59-64.

Göritz, A.S. (2006) 'Incentives in web studies: methodological issues and a review', International Journal of Internet Science, 1 (1): 58-70.

Groves, R.M. (1978) 'An empirical comparison of two telephone sample designs', Journal of Marketing Research, 15 (4), 622-31.

Groves, R.M. (1989) Survey Errors and Survey Costs. New York: John Wiley.

Internet World Stats (2005) Accessed online at www.internetworldstats.com/top25.htm on April 23, 2007.

Jackob, N., Arens, J. and Zerback, T. (2005) 'Sampling procedure, questionnaire design, online implementation, and survey response in a multinational online journalist survey'. Paper presented at the Joint WAPOR/ISSC Conference: Conducting International Social Surveys. Accessed online at ciss.
ris.org/uploadi/editor/1132070316WAPORPaper.pdf on September 30, 2006.

Kish, Leslie (1965) Survey Sampling. New York: Wiley-Interscience. (New edition February 1995).

Krishnamurthy, S. (2002) 'The ethics of conducting e-mail surveys'. Readings in virtual research ethics: issues and controversies, accessed online at ssrn.com/ abstract_id=651841 on September 30, 2006.

Kypri, K. and Gallagher, S.J. (2003) 'Incentives to increase participation in an Internet survey of alcohol use: a controlled experiment', Alcohol and Alcoholism, 38 (5): 437-441.

Lockett, A. and Blackman, I. (2004) 'Conducting market research using the Internet: the case of Xenon Laboratories', Journal of Business and Industrial Marketing, 19 (3), 178-87.

Midwest Book Review (2003) 'Book review: Take advantage of the Internet and preserve data integrity', accessed online at http://www.amazon. com/Conducting-Research-Surveys-E-Mail-Web/dp/ $0833031104 /$ sr=1-3/qid=1160191573/ref=sr_1_3/ 102-5971652-1424906?ie=UTF8\&s=books on October 6, 2006.

Patton, M.Q. (2002) Qualitative Evaluation and Research Methods, London: Sage.

Pineau, V. and Dennis, J.M. (2004) 'Methodology for probability-based recruitment for a web-enabled panel', dated November 21, 2004. Downloaded from www.knowledgenetworks.com/ganp/reviewerinfo.html on July 21, 2006.

Ross, I. (1977) The Loneliest Campaign: The Truman Victory, (reprint edition). Greenwood Press. Accessed online at www.trumanlibrary.org/whistlestop/study_ collections/1948campaign/large/docs/documents/ index. php? documentdate $=1968-00-00 \&$ documentid= $53 \&$ studycollectionid=Election\&pagenumber $=1$ on October 1, 2006.

Schonlau, Matthias, Fricker, Jr. Ronald D. and Elliott, Marc N. (2002) Conducting Research Surveys via E-Mail and the Web, MR-1480-RC, Santa Monica: RAND.

Schonlau, Matthias, Zapert, K., Simon, L.P., Sanstad, K.H., Marcus, S.M., Adams, J., Spranca, M., Kan, H., Turner, R. and Berry, S.H.. (2004) 'A comparison between responses from a propensityweighted web survey and an identical RDD survey', Social Science Computer Review, 22 (1): 128-38.

Sheehan, K.B. (1999) 'Using e-mail to survey Internet users in the United States: methodology and assessment', Journal of Computer Mediated Communication, (4) 3. Accessed online at http://jcmc.indiana. edu/vol14/issue3/sheehan.html on July 6, 2006.

Shillewaert, N., Langerak, F. and Duhamel, T. (1998) 'Non-probability sampling for WWW 
surveys: a comparison of methods', Journal of the Market Research Society, 40 (4): 307-22.

Siah, C.Y. (2005) 'All that glitters is not gold: examining the perils and obstacles in collecting data on the Internet', International Negotiation, 10: $115-30$.

Soetikno, R.M., Provenzale, D. and Lenert, L.A. (1997) 'Studying ulcerative colitis over the World Wide Web', American Journal of Gastroenterology, 92 (3): 457-60.

Squires, P. (1988) 'Why the 1936 "Literary Digest" poll failed', The Public Opinion Quarterly, 53 (1): 125-33.

Witte, J.C., Amoroso, L.M. and Howard, P.E.N. (2000) 'Research methodology: method and representation in Internet-based survey tools - mobility, community, and cultural identity in Survey2000', Social Sciences Computer Review, 18 (2): 179-95.

Wright, T. and Tsao, H.J. (1983) 'A frame on frames: an annotated bibliography'. In T. Wright (ed.) Statistical Methods and the Improvement of Data Quality. New York: Academic Press.

Zetterberg, H.L. (2004) 'US election 1948: the first great controversy about polls, media, and social science'. Paper presented at the WAPOR regional conference on 'Elections, News Media and Public Opinion' in Pamplona, Spain, November 24-26. Accessed online at http://www.zetterberg.org/Lectures//041115.htm on October 1, 2006.

\section{FURTHER READING}

Mail and Internet Surveys: The Tailored Design Method by Dillman (2007 edition) and Survey Errors and Survey Costs by Groves (1989). Each of these texts focuses on the entire process of designing and fielding surveys, not just sampling.

Conducting Research Surveys via E-Mail and the Web by Schonlau, Fricker and Elliott (2002) 'is a practical and accessible guide to applying the pervasiveness of the Internet to the gathering of survey data in a much faster and significantly less expensive manner than traditional means of phone or mail communications.' (Midwest Book Review, 2003)

'Review: Web Surveys: A Review of Issues and Approaches' by Mick P. Couper, published in The Public Opinion Quarterly, is an excellent and highly cited article that emphasizes many of the points and ideas discussed in this chapter. It also provides additional examples to those presented in this chapter.

Sampling Techniques by Cochran (1977) is one of the classic texts on the mathematical details of survey sampling, covering a wide range of sampling methods applicable to all types of survey effort. 